\title{
EFFECT OF NITROGEN AND PHOSPHOROUS FERTILIZATION ON SOME WHEAT CULTIVARS PRODUCTIVITY
}

\author{
M. M. El-Balasy (1), A. A. El-Hosary'(2), G. Y. Hammam ${ }^{(2)}$, \\ S. A. Allam ${ }^{(2)}$, R. B. Abo-Arab ${ }^{(1)}$, E. M. El-Gedwy ${ }^{(2)}$ and A. A. El-Hosary ${ }^{(2)}$ \\ (1) Plant protection Res. Inst., Agric. Res. Center, Dokki, Giza, Egypt. \\ (2) Agronomy Department, Faculty of Agriculture, Benha University
}

Received: Feb. 6, 2017

Accepted: Mar. 5, 2017

\begin{abstract}
Two field experiments were carried out at Sidi Salem Directorate, Kafr ELSheikh Governorate, Egypt, during the two successive winter seasons of 2011/2012 and 2012/2013 to study the effect of four nitrogen fertilization rates $(0,37.5,75$ and $112.5 \mathrm{~kg} \mathrm{~N} / \mathrm{fed}$ ) and three phosphorous fertilization rates $\left(0,15\right.$ and $\left.30 \mathrm{~kg} \mathrm{P}_{2} \mathrm{O}_{5} / \mathrm{fed}\right)$ on the growth characters, yield, its component and some chemical properties of three bread wheat cultivars (Gemmeiza 9, Sakha 93 and Giza 168). The experimental design was split split plot design in four replications. The obvious results of this investigation can be summarized as follows:

All traits of wheat under study, i.e. No. of tillers $/ \mathrm{m}^{2}$, No. of spike $/ \mathrm{m}^{2}$, plant height, spike length, No. of kernels/spike, 1000-kernel weight, kernels weight/spike, straw yield/fed, grain yield/fed, biological yield/fed, harvest index, kernels nitrogen content (\%) and grain protein yield/fed showed significantly increased by increasing nitrogen fertilizer rates, except kernels phosphorous content (\%). Application of $112.5 \mathrm{~kg} \mathrm{~N} / \mathrm{fed} \mathrm{significantly} \mathrm{gave} \mathrm{the} \mathrm{maximum} \mathrm{values}$ of all traits. Increasing phosphorous fertilizer rates up to $30 \mathrm{Kg} \mathrm{P}_{2} \mathrm{O}_{5} / \mathrm{fed}$ significantly increased No. of tillers $/ \mathrm{m}^{2}$, No. of spike $/ \mathrm{m}^{2}$, plant height, spike length, straw yield/fed, grain yield/fed, biological yield/fed and kernels phosphorous content (\%) in the two seasons. While, No. of kernels/spike, 1000-kernel weight, kernels weight/spike, harvest index, kernels nitrogen content $\%$ and grain protein yield/fed were not affected by increasing phosphorous fertilizer rates. The results showed that wheat cultivars were significantly differed in all traits studied under study in the both seasons, except biological yield kg/fed, kernels nitrogen content (\%) and kernels phosphorous content (\%). Gemmeiza 9 cultivar significantly surpassed Sakha 93 and Giza 168 cultivars in plant height, spike length, 1000-kernel weight, kernels weight/spike, grain yield/fed, harvest index and kernels protein yield/fed in the both seasons, while, Sakha 93 recorded the highest values of No. of kernels/spike. Morever, Giza 168 surpassed the other two cultivars in No. of tillers $/ \mathrm{m}^{2}$, No. of spike $/ \mathrm{m}^{2}$ and straw yield/fed. The first order interactions between $(112.5$ $\mathrm{kg} \mathrm{N} / \mathrm{fed} X 30 \mathrm{Kg} \mathrm{P} \mathrm{P}_{5} / \mathrm{fed}$ ), (112.5 $\mathrm{kg} \mathrm{N} /$ fed X Gemmeiza 9) and (30 $\mathrm{Kg} \mathrm{P}_{2} \mathrm{O}_{5} / \mathrm{fed} X$ Gemmeiza 9) as well as the second order interaction between $112.5 \mathrm{~kg} \mathrm{~N} / \mathrm{fed} X 30 \mathrm{Kg} P_{2} \mathrm{O}_{5} / \mathrm{fed} X$ Gemmeiza 9 were significantly recorded the greatest grain yield/fed as compared with the others interactions in the both seasons. It could be summarized that sown wheat Gemmeiza 9 cultivar and fertilization by $30 \mathrm{Kg} \mathrm{P}_{2} \mathrm{O}_{5}+112.5 \mathrm{~kg} \mathrm{~N} /$ fed maximized grain yield per unit area.
\end{abstract}

Key words: Wheat cultivars, phosphorous fertilization and nitrogen fertilization.

\section{INTRODUCTION}

Bread wheat (Triticum aestivum, L.) is the most important cereal crops in Egypt as well as over the world and covers more of the earth's surface, used in human food and animal feed. It is a staple food for more than one third of the world population. In Egypt, wheat provides $37 \%$ of the total calories for the people and $40 \%$ of the protein in the Egyptian diet (Min. Agric. Statistic Year Book, 2000). The total production of wheat was 9.61 million tons in 2015 produced from an area of 3,468,864 faddans, (Bull. Agric. Stat., 2016), with an average yield of 2880 $\mathrm{kg} / \mathrm{fad}$. The total consumption of wheat nearly from 16.26 million tons, thus the local 
production covers more than $59.09 \%$ of the local consumption. Consequently, increasing wheat production during the last period became one of the most important goals of the Egyptian government to fulfill the food security for the people. This target can be achieved through expanding wheat area in the delta and along the valley as well as in the new reclaimed lands and rainfed areas, or by means of raising the yield through growing high yielding cultivars and applying the optimum cultural practices.

Determination the required rate of nitrogen fertilization of wheat is of the main important practices of great contribution for the highest production of better quality, as well as nitrogen is a key element for wheat productivity as well as in many other field cereal crops. Several investigations reported that increasing nitrogen fertilization rates caused significant increase in growth, yield, its components and chemical properties traits of wheat. Shafshak et al., 2003; Allam 2005; Abd El-Maaboud et al., 2006; AlAfandy et al., 2007; Khan et al., 2008; Benin et al., 2012; Gomaa et al., 2012; Hafez et al., 2012; Zaki et al., 2012; Ashmawy et al., 2013; Noureldin et al., 2013; Sultana et al., 2013; Khalid et al., 2014; El-Hosary et al., 2015, Jelic et al., 2015 and Mehasen et al., 2015 showed that increases in No. of tillers $/ \mathrm{m}^{2}$, No. of spike $/ \mathrm{m}^{2}$, plant height, spike length, No. of kernels/spike, 1000-kernel weight, kernels weight/spike, straw yield/fed, grain yield/fed, biological yield/fed, harvest index, kernels nitrogen content $\%$ and grain protein yield/fed with increasing nitrogen fertilization rates.

Phosphorous is the second major nutrient that produces significant increment in wheat growth and productivity if added in appreciated dosage as a fertilizer. It is very important for most of the physiological processes of plant growth and metabolism. Abdul Galil et al., 2003; Shafshak et al., 2003; Abd El-Maaboud et al., 2006; AlAfandy et al., 2007 Khan et al., 2008; and
Jelic et al., 2015 indicated that application of phosphorous fertilization at the perfect time and quantity increased significantly wheat traits including No. of tillers $/ \mathrm{m}^{2}$, No. of spike $/ \mathrm{m}^{2}$, plant height, spike length, straw yield/fed, grain yield/fed, biological yield/fed and kernels phosphorous content \%.

Several investigators showed that wheat cultivars differed in growth, yield, its components and some chemical properties (Abdul Galil et al., 2003; Abd El-Maaboud et al., 2006; Benin et al., 2012; Gomaa et al., 2012; Hafez et al., 2012; Zaki et al., 2012; Ashmawy et al., 2013; Noureldin et al., 2013; Sultana et al., 2013; Khalid et al., 2014; El-Hosary et al., 2015, Jelic et al., 2015 and Mehasen et al., 2015).

The aim of this investigation was designed to study the effect of four nitrogen and three phosphorous fertilization rates on growth, yield, yield components and chemical composition in three wheat cultivars.

\section{MATERIALS AND METHODS}

Two field experiments were carried out at Sidi Salem Directorate, Kafr El-Sheikh Governorate, Egypt, during the two successive winter seasons of 2011/2012 and $2012 / 2013$. The aim of this study was to investigate the effect of four nitrogen and three phosphorous fertilization levels on the growth traits, yield, its components and some chemical properties of three bread wheat cultivars. Soil texture of the experimental site was clay and salty of $\mathrm{pH}$ nearly of 8.00 . The physical and chemical properties of the experimental soil were determined according to the standard procedures described by Black, 1965) and represented in Table, 1 in each of the two growing seasons.

Each experiment included 36 treatments which were the combination of four nitrogen fertilizer rates, three phosphorous fertilizer rates and three bread wheat cultivars. 
Table 1: Physical and chemical properties of the experimental soil units of the two growing seasons (2011/2012 and 2012/2013).

\begin{tabular}{|c|c|c|}
\hline \multirow{2}{*}{ Properties } & \multicolumn{2}{|c|}{ Seasons } \\
\hline & 2011/2012 & 2012/2013 \\
\hline \multicolumn{3}{|l|}{ Chemical analysis } \\
\hline E.C. & 4.85 & 5.02 \\
\hline $\mathrm{pH}(1: 2.5)$ & 7.84 & 8.12 \\
\hline $\mathrm{N}$ (ppm) (available) & 32 & 39 \\
\hline P (ppm) (available) & 7.83 & 8.15 \\
\hline \multicolumn{3}{|c|}{ Soluble cations and anions ( ppm ) } \\
\hline $\mathrm{Na}^{+}$ & 30.4 & 31 \\
\hline $\mathrm{Ca}^{2+}$ & 10.60 & 10.60 \\
\hline $\mathrm{Mg}^{2+}$ & 6.70 & 7.78 \\
\hline $\mathrm{K}^{+}$ & 0.80 & 0.90 \\
\hline $\mathrm{HCO}_{3}^{-}$ & 3.50 & 4.50 \\
\hline $\mathrm{Cl}^{-}$ & 23.25 & 26.58 \\
\hline $\mathrm{SO}_{4}^{2-}$ & 21.75 & 19.20 \\
\hline \multicolumn{3}{|c|}{ Particle size distribution ( mechanical analysis ) } \\
\hline Course sand \% & 4. 98 & 5.32 \\
\hline Find sand \% & 15.76 & 16. 18 \\
\hline Silt \% & 24.61 & 26. 29 \\
\hline Clay \% & 54.65 & 52. 21 \\
\hline Texture grade & Clay & Clay \\
\hline
\end{tabular}

Factors under study were as follows:

1- Four nitrogen fertilization rates, i.e. 0 , $37.5,75$ and $112.5 \mathrm{~kg} \mathrm{~N} / \mathrm{fed}$, nitrogen fertilizer was applied in form of urea (46 $\% \mathrm{~N}$ ), and divided into two equal parts and applied before the first and second irrigations in each season.

2- Three phosphorous fertilization rates, i.e. 0,15 and $30 \mathrm{~kg} \mathrm{P}_{2} \mathrm{O}_{5} / \mathrm{fad}$, phosphorous fertilizer was applied in form of calcium super phosphate $\left(12.5 \% \mathrm{P}_{2} \mathrm{O}_{5}\right)$, and applied during soil preparation in the two seasons.

3- Three bread wheat cultivars namely, Gemmeiza 9, Sakha 93 and Giza 168. Seeds of the three wheat cultivars were secured every two seasons from Field Crop Institute, Sakha Agriculture Research Station, Agricultural Research Center (ARC).

The experimental design was split split plot design (Gomez and Gomez, 1984) in four replications. The four nitrogen fertilizer rates were random distributed in the main plots, whereas the three phosphorous fertilizer rates were arranged at random in sub plots and the three cultivars of wheat were assigned in random in sub sub plots. The preceding summer crop in the two seasons was rice. The sub sub plot size was $10.5 \mathrm{~m}^{2}$ of $3.0 \times 3.5 \mathrm{~m}$ including 15 rows 20 $\mathrm{cm}$ apart and 3.5 meter length. Seeding rate 
for the three wheat cultivars was $60 \mathrm{~kg}$ seeds/fed. Experiments were planted on November $16^{\text {th }}$ and $20^{\text {th }}$ of in the first season (2011/2012) and the second season (2012/2013), respectively. The other recommended agronomic practices of growing wheat were applied in the manner prevailing in the region were practiced.

\section{Studied parameters: The following growth characters, yield and yield attributes were recorded:}

At harvest, all plants in one square meter from each sub sub plot were harvested to determine No. of tillers $/ \mathrm{m}^{2}$, No. of spikes $/ \mathrm{m}^{2}$ and 1000-kernel weight (g). Then, ten fertile tillers from the previous one square meter were chosen randomly to estimate the plant height $(\mathrm{cm})$, spike length $(\mathrm{cm})$, No. of kernels/spike and kernels weight/spike (g). While, biological, grain and straw yields $(\mathrm{kg} / \mathrm{fed})$ and harvest index \% were estimated from the whole sub sub plot yield.

\section{Chemical analysis}

Wheat grain samples were taken after harvest at random from kernels of ten spikes to determine kernels nitrogen content (\%) according to the modified micro Kjeldahl method as described by A. O. A. C., 1980, kernels phosphorous content (\%) was determined by Spectrophotometer as reported by (Jackson 1973). While, protein yield/fed was calculated by multiplying kernels nitrogen content $X 5.7 \times$ grain yield $\mathrm{kg} / \mathrm{fed}$.

\section{Statistical analysis:}

The analysis of variance was carried out according to the procedure described by Gomez and Gomez (1984). Data were statistically analyzed according to using the MSTAT-C Statistical Software Package (Michigan State University, 1983). Where the F-test showed significant differences among means $L$. S. D. test at 0.05 level was used to compare between means.

\section{RESULTS AND DISCUSSION}

\section{Effect of nitrogen fertilization:}

Results in Tables 2 and 3 showed that growth, yield, its attributes and chemical properties of wheat, i.e. No. of tillers $/ \mathrm{m}^{2}$, No. of spikes $/ \mathrm{m}^{2}$, plant height $(\mathrm{cm})$, spike length $(\mathrm{cm})$, No. of kernels/spike, kernels weight/spike (g), 1000-kernel weight (g), biological yield $(\mathrm{kg} / \mathrm{fed})$, grain yield $(\mathrm{kg} / \mathrm{fed})$, straw yield $(\mathrm{kg} / \mathrm{fed})$, harvest index (\%), kernels nitrogen content $\%$ and kernels protein yield $(\mathrm{kg} / \mathrm{fed})$ were significantly increased by increasing nitrogen fertilizer rates up to $112.5 \mathrm{~kg} \mathrm{~N} / \mathrm{fed}$ in 2011/2012 and 2012/2013 seasons. While, kernels phosphorous content \% was not significant. In general, the higher nitrogen rate $(112.5 \mathrm{~kg}$ $\mathrm{N} / \mathrm{fed}$ ) was more effective in increasing values of all studied traits, also, produced the maximum grain yield/fed and proved significantly superior to other nitrogen rates. The nitrogen treatments of 37.5, 75 and $112.5 \mathrm{~kg} \mathrm{~N} / \mathrm{fed}$ significantly increased grain yield/fed of wheat by $44.68,69.29$ and 84.26 $\%$ in the first season, respectively, and by $43.08,67.99$ and $80.89 \%$ in the second season, respectively when compared to without nitrogen added. The present results clearly indicate that nitrogen application induced increases in growth, yield and yield components traits of wheat showing the major role of this vital nutritive element. The increase in nitrogen application encourages photosynthesis activities and the metabolic efficiency as well as promoting the cell division, vegetative growth and encouraging the juvenility and active persistence of meristimatic tissues which contributes in enhancing the accumulation of the produced metabolites of wheat. Many investigators came out with similar results as Shafshak et al., 2003; Allam 2005; Abd El-Maaboud et al., 2006; Al-Afandy et al., 2007; Khan et al., 2008; Benin et al., 2012; Gomaa et al., 2012; Hafez et al., 2012; Zaki et al., 2012; Ashmawy et al., 2013; Noureldin et al., 2013; Sultana et al., 2013; Khalid et al., 2014; El-Hosary et al., 2015, Jelic et al., 2015 and Mehasen et al., 2015. 


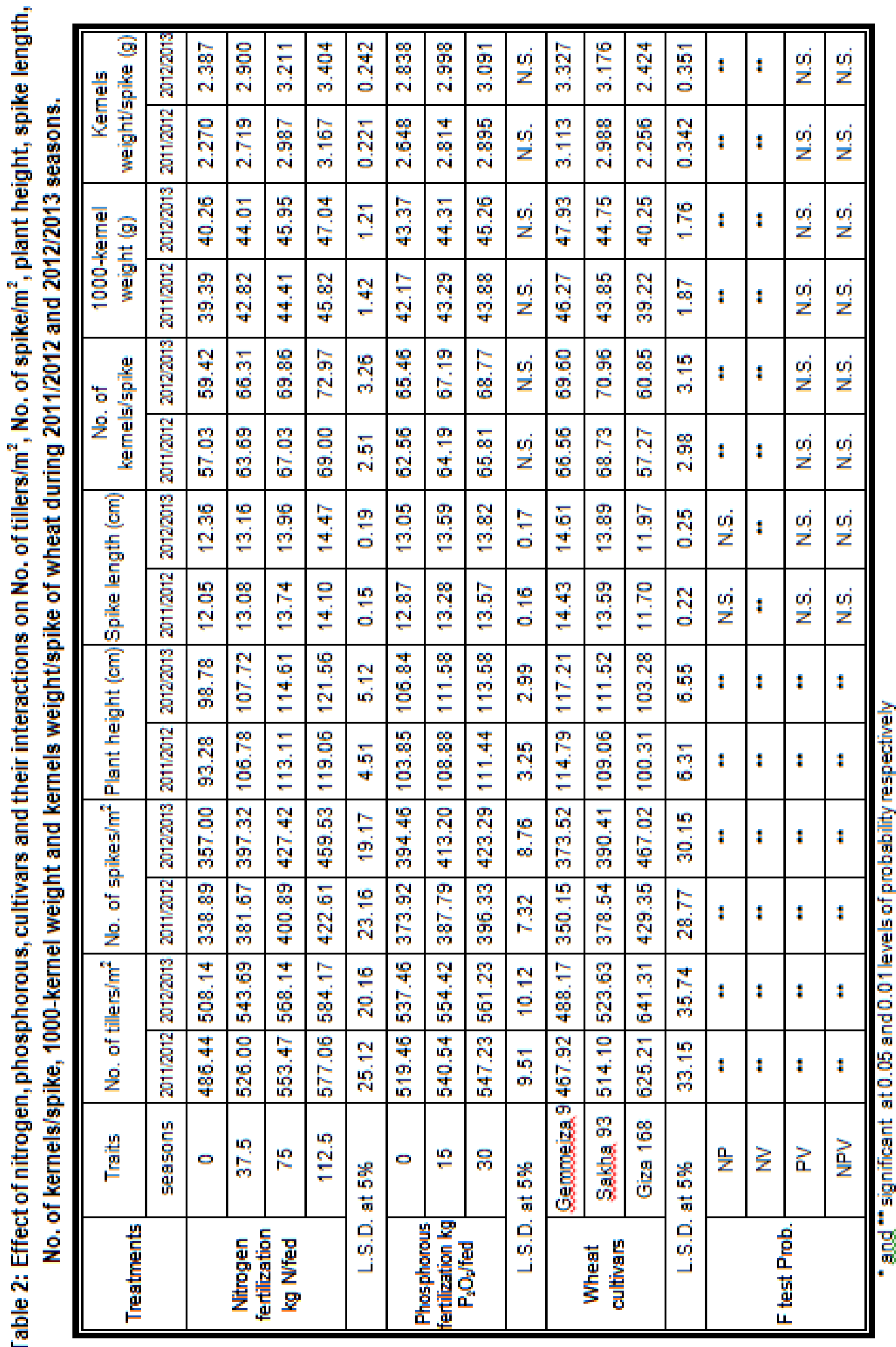




\begin{tabular}{|c|c|c|c|c|c|c|c|c|c|c|c|c|c|c|c|c|c|c|}
\hline 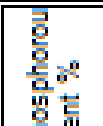 & 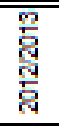 & 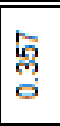 & 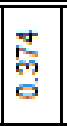 &  & 点 & 家 & 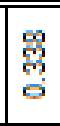 & 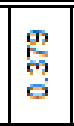 & 桨 & 哭 & \begin{tabular}{|l} 
总 \\
0 \\
0 \\
0
\end{tabular} &  & 竎 & $\begin{array}{c}j \\
\dot{j} \\
z\end{array}$ & $\frac{j}{\dot{o}}$ & $\begin{array}{l}j \\
\dot{j} \\
z\end{array}$ & $\begin{array}{l}\dot{j} \\
z \\
z\end{array}$ & $\begin{array}{l}\text { oj } \\
z\end{array}$ \\
\hline 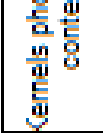 &  &  &  & 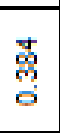 & 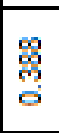 & $\begin{array}{l}\dot{j} \\
z \\
z\end{array}$ & 意 & 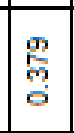 & 旁 & 훙 & 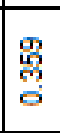 & 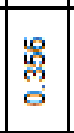 & $\begin{array}{l}\frac{7}{\sigma} \\
\dot{\sigma} \\
0\end{array}$ & $\begin{array}{l}j o j \\
z \\
z\end{array}$ & $\begin{array}{l}j \\
\dot{j} \\
z\end{array}$ & $\begin{array}{l}j \\
\dot{j o j} \\
z\end{array}$ & $\begin{array}{l}\dot{j} \\
\dot{j} \\
z\end{array}$ & ioj \\
\hline 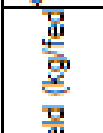 & 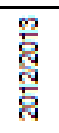 & 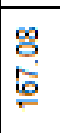 & 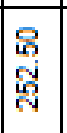 & $\begin{array}{l}\bar{\infty} \\
\tilde{d} \\
\bar{d}\end{array}$ & $\frac{8}{\mathrm{j}}$ & 点 & 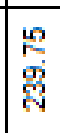 & 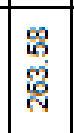 & 芯 & ij &  & $\begin{array}{l}\text { ले } \\
\text { हु. } \\
\end{array}$ & $\begin{array}{l}\text { 울 } \\
\text { 枳 }\end{array}$ & 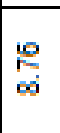 & : & : & : & : \\
\hline  & 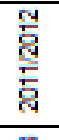 &  &  & $\begin{array}{l}g \\
\stackrel{g}{\mathrm{~N}} \\
\mathrm{~N}\end{array}$ & $\overline{\bar{c}}$ & 竎 & 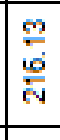 & \begin{tabular}{|l|}
\multicolumn{2}{c}{} \\
产 \\
\end{tabular} & 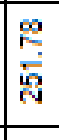 & $\frac{j o j}{z}$ & $\begin{array}{l}g \\
8 \\
\text { 总 } \\
\end{array}$ & 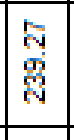 & 芯 & $\begin{array}{c}8 \\
0 \\
\infty\end{array}$ & : & : & : & : \\
\hline 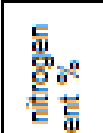 & 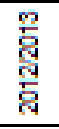 & 总 & 兽 & 鄀 & 芯 & 荢 & 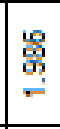 & 늘 & 跑 & $\begin{array}{c}j i \\
z \\
z\end{array}$ & 焉 & 兽 & 옹 & $\begin{array}{l}j \\
\dot{j} \\
z\end{array}$ & $\stackrel{j}{i j}$ & $\begin{array}{l}j \\
\dot{\omega} \\
z\end{array}$ & $\begin{array}{l}j \dot{j} \\
z \\
z\end{array}$ & oj \\
\hline 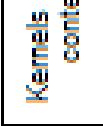 &  & 啚 & 荵 & 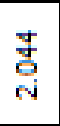 & 跑 & 훙 & 志 & ס̊ & ర్ & $\begin{array}{l}j \\
z \\
z\end{array}$ & 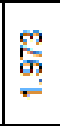 & 兽 & 员 & $\begin{array}{l}j \\
\dot{j o j} \\
z\end{array}$ & $\begin{array}{l}j \\
\dot{j} \\
z\end{array}$ & $\begin{array}{l}j \\
\dot{j} \\
z\end{array}$ & $\begin{array}{l}j \dot{j} \\
z \\
z\end{array}$ & ioj \\
\hline & 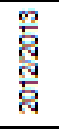 & ్ㅗㅇ & $\underset{\infty}{\infty}$ & \begin{tabular}{|l|} 
\\
总 \\
g.
\end{tabular} & $\begin{array}{l}\text { E } \\
\text { o }\end{array}$ & 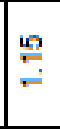 & 誉 & $\begin{array}{l}\mathrm{c} \\
\text { gi }\end{array}$ & 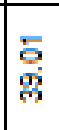 & $\begin{array}{l}o j \\
z \\
z\end{array}$ & 离 & \begin{tabular}{|l|} 
\\
0 \\
g. \\
g
\end{tabular} & 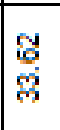 & $\mathscr{B}$ & $\begin{array}{c}\text { oj } \\
z\end{array}$ & : & : & ơ \\
\hline & 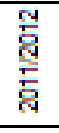 & 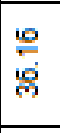 & 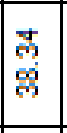 & $\begin{array}{l}\text { 总 } \\
\text { g } \\
g\end{array}$ & 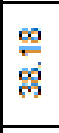 & $\cong$ & $\begin{array}{l}3 \\
\text { ले } \\
5\end{array}$ & $\begin{array}{l}\text { 年 } \\
\text { 尊 }\end{array}$ & \begin{tabular}{|l}
$\bar{i}$ \\
$\overline{0}$ \\
o
\end{tabular} & oj & \begin{tabular}{|l}
$\stackrel{2}{2}$ \\
\multirow{2}{*}{} \\
v
\end{tabular} & \begin{tabular}{|l|}
$\bar{\sigma}$ \\
$\dot{\sigma}$ \\
$\bar{\sigma}$
\end{tabular} & $\begin{array}{l}\text { 志 } \\
\text { d्ల }\end{array}$ & $\stackrel{i n g}{N}$ & oj & : & : & ơ \\
\hline 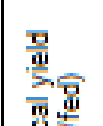 & 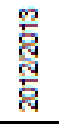 & $\begin{array}{l}\text { g } \\
\text { ơ } \\
\text { 委 } \\
\end{array}$ & 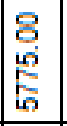 & $\begin{array}{l}9 \\
0 \\
0 \\
0 \\
8 \\
0\end{array}$ & 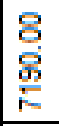 &  & 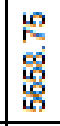 & 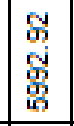 & 閭 & 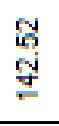 & 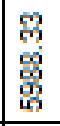 & 它 & 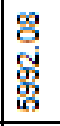 & $\begin{array}{l}0 \\
z\end{array}$ & : & : & : & : \\
\hline  & 米 & 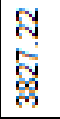 &  & 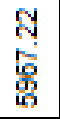 & $\begin{array}{l}9 \\
0 \\
0 \\
58 \\
8 \\
\end{array}$ &  &  & 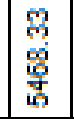 & 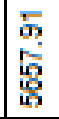 & 号 & 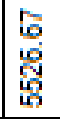 & 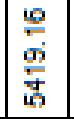 & 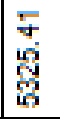 & $\begin{array}{l}\infty \\
z \\
z\end{array}$ & : & : & : & : \\
\hline 总 & 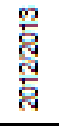 & 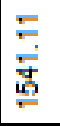 & \begin{tabular}{|l|}
8 \\
总 \\
त̂
\end{tabular} & 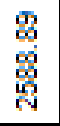 & 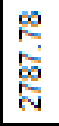 &  & 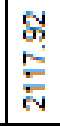 & 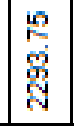 &  & $\begin{array}{l}\overline{5} \\
\text { 通 }\end{array}$ &  & 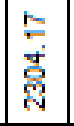 & 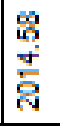 & 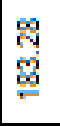 & : & : & : & : \\
\hline & 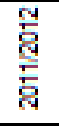 &  & \begin{tabular}{|l|} 
Na \\
జू. \\
\end{tabular} & 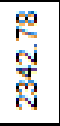 & 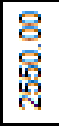 & 물 & 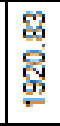 & 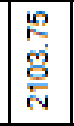 & 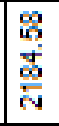 & $\begin{array}{l}\text { 岢 } \\
\text { ơ⿱ }\end{array}$ & 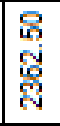 & 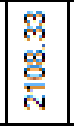 &  &  & : & : & : & : \\
\hline 要票 & 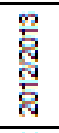 & 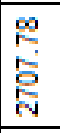 & \begin{tabular}{|l|}
8 \\
\\
\\
s. \\
\end{tabular} & \begin{tabular}{|l|}
8 \\
0 \\
⿳亠口子丸 \\
\end{tabular} & \begin{tabular}{|l|}
\multirow{2}{*}{} \\
竎 \\
\end{tabular} & $\begin{array}{l}\overline{\bar{n}} \\
\tilde{\mathrm{N}} \\
\tilde{N}\end{array}$ & 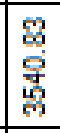 & \begin{tabular}{|l|r|} 
兽 \\
总 \\
\end{tabular} & 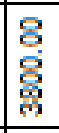 &  & 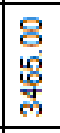 & 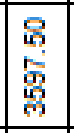 & 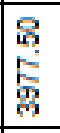 & $\begin{array}{l}\text { 志 } \\
\text { ज्ञ } \\
\text { गे }\end{array}$ & $:$ & : & : & : \\
\hline 袋 & 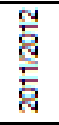 & 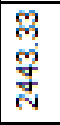 & 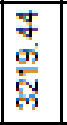 & 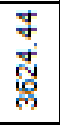 & 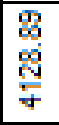 &  &  &  &  & $\begin{array}{l}\frac{10}{2} \\
\frac{10}{5}\end{array}$ & 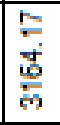 & 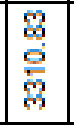 & \begin{tabular}{|l|}
8 \\
总 \\
絗 \\
\end{tabular} & 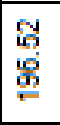 & : & : & : & : \\
\hline 袁 & 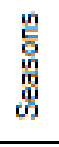 & 0 &  & $\stackrel{\text { g }}{2}$ & 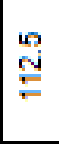 & $\because 8$ & 0 & œ & হি & 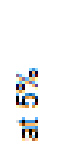 & 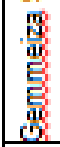 & 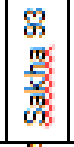 & 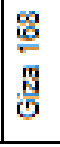 &  & $\frac{a}{z}$ & z & z & $\frac{a}{2}$ \\
\hline 常 & & & 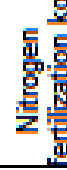 & & & 送 & &  & & ن & & 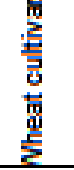 & & ن & & & & \\
\hline
\end{tabular}




\section{Effect of phosphorous fertilization:}

Results of the effect of phosphorous application on yield of wheat and its related characters are shown in Tables 2 and 3 . The results indicate that increasing $P$ fertilizer rates from 0 to $30 \mathrm{~kg} \mathrm{P}_{2} \mathrm{O}_{5} / \mathrm{fed}$ increased significantly No. of tillers $/ \mathrm{m}^{2}$, No. of spikes $/ \mathrm{m}^{2}$, plant height $(\mathrm{cm})$, spike length $(\mathrm{cm})$, biological yield $(\mathrm{kg} / \mathrm{fed})$, grain yield $(\mathrm{kg} / \mathrm{fed})$, straw yield $(\mathrm{kg} / \mathrm{fed})$ and kernels phosphorous content \% in the both seasons. In general, the higher phosphorous fertilizer rate $\left(30 \mathrm{~kg} \mathrm{P}_{2} \mathrm{O}_{5} / \mathrm{fed}\right)$ was more effective in increasing values of all studied traits, also, produced the greatest grain yield/fed and proved significantly superior to other phosphorous fertilizer rates. The phosphorous fertilizer treatments of 15 and $30 \mathrm{~kg} \mathrm{P}_{2} \mathrm{O}_{5} / \mathrm{fed}$ significantly increased grain yield/fed of wheat by 9.52 and $13.73 \%$ in the first season, respectively, and by 8.30 and $14.76 \%$ in the second season, respectively when compared to without phosphorous fertilizer added. These results showed the importance of $P$ as an important nutritive element for wheat. Similar results were also obtained by Abdul Galil et al., 2003; Shafshak et al., 2003; Abd ElMaaboud et al., 2006; Al-Afandy et al., 2007 Khan et al., 2008; and Jelic et al., 2015. The results in Table 2 and 3 also show that No. of kernels/spike, kernels weight/spike (g), 1000-kernel weight $(\mathrm{g})$, harvest index (\%), kernels nitrogen content $\%$ and kernels protein yield $(\mathrm{kg} / \mathrm{fed})$ showed no significant change.

\section{Effect of wheat cultivars:}

Results presented in Tables 2 and 3 revealed that the differences between the studied three cultivars, i.e. Gemmeiza 9, Sakha 93 and Giza 168 in all growth traits, yield, its components and chemical properties in the both seasons were significant except, biological yield $\mathrm{kg} / \mathrm{fed}$, kernels nitrogen content $\%$ and kernels phosphorous content \% were not significant. These results revealed that Gemmeiza 9 cultivar recorded the greatest values of plant height (114.79 and $117.21 \mathrm{~cm})$, spike length $(14.43$ and $14.61 \mathrm{~cm}), 1000$-kernel weight (46.27 and $47.93 \mathrm{~g}$ ), kernels weight/spike (3.113 and 3.327), grain yield (2362.50 and $2523.33 \mathrm{~kg} / \mathrm{fed}$ ), harvest index (42.75 and $42.14 \%)$ and kernels protein yield (265.69 and $284.64 \mathrm{~kg}$ /fed) in the first and second seasons, respectively. The three tested cultivars could be arranged in a descending order with regard to the previous traits as follows, Gemmeiza 9, Sakha 93 and Giza 168. However, results may reveal the superiority of Sakha 93 cultivar in No. of kernels/spike (68.73 and 70.96 kernels) in the first and second seasons, respectively. Moreover, Giza 168 cultivar gave the highest values of No. of tillers $/ \mathrm{m}^{2} \quad(625.21$ and 641.31 tillers), No. of spikes $/ \mathrm{m}^{2}$ (429.35 and 467.02 spikes) and straw yield (3587.08 and $3977.50 \mathrm{~kg} / \mathrm{fed}$ ) in the first and second seasons, respectively. These differences may be due to the genetic differences between the three cultivars. Also, the differences in 1000-kernel weight might be attributed to the variation in translocation rate of photosynthetic from leaves to the storing organs, i.e. the grain. The superiority of Gemmeiza 9 cultivar in grain yield over the other wheat cultivars might be due to the increase in growth and yield components, namely, plant height, spike length, kernels weight/spike, 1000-kernel weight. These results are in harmony with those reported by Abdul Galil et al., 2003; Abd El-Maaboud et al., 2006; Benin et al., 2012; Gomaa et al., 2012; Hafez et al., 2012; Zaki et al., 2012; Ashmawy et al., 2013; Noureldin et al., 2013; Sultana et al., 2013; Khalid et al., 2014; El-Hosary et al., 2015, Jelic et al., 2015 and Mehasen et al., 2015.

\section{Interactions effect: \\ The interaction effect between nitrogen and phosphorous fertilization rates:}

Data in Tables, 2 and 3 showed that the interaction between nitrogen and phosphorous fertilization rates were significant on No. of tillers $/ \mathrm{m}^{2}$, No. of 
spikes $/ \mathrm{m}^{2}$, plant height $(\mathrm{cm})$, No. of kernels/spike, kernels weight/spike (g), 1000-kernel weight (g), biological yield $(\mathrm{kg} / \mathrm{fed})$, grain yield $(\mathrm{kg} / \mathrm{fed})$, straw yield $(\mathrm{kg} / \mathrm{fed})$ and kernels protein yield $(\mathrm{kg} / \mathrm{fed})$. The results illustrated in Table, 4 showed that the highest values of biological yield (6873.33 and $7390.00 \mathrm{~kg} / \mathrm{fed})$, straw yield (4253.33 and $4516.67 \mathrm{~kg} / \mathrm{fed}$ ), grain yield (2620.00 and $2873.33 \mathrm{~kg} / \mathrm{fed}$ ) and kernels protein yield $(311.08$ and $343.12 \mathrm{~kg} / \mathrm{fed}$ ) were recorded from wheat plants which fertilized by $112.5 \mathrm{~kg} \mathrm{~N}$ and $30 \mathrm{~kg} \mathrm{P}_{2} \mathrm{O}_{5} / \mathrm{fed}$. Such results are in accordance with those obtained by Shafshak et al., 2003; Abd ElMaaboud et al., 2006; Al-Afandy et al., 2007 Khan et al., 2008; and Jelic et al., 2015.

\section{The interaction effect between nitrogen fertilizer rates and wheat cultivars:}

Significant effect of the interaction between nitrogen fertilizer rates and wheat cultivars obtained for almost growth, yield and yield components of wheat namely, No. of tillers $/ \mathrm{m}^{2}$, No. of spikes $/ \mathrm{m}^{2}$, plant height $(\mathrm{cm})$, spike length $(\mathrm{cm})$, No. of kernels/spike, kernels weight/spike $(\mathrm{g}), 1000$-kernel weight $(\mathrm{g})$, biological yield $(\mathrm{kg} / \mathrm{fed})$, grain yield $(\mathrm{kg} / \mathrm{fed})$, straw yield $(\mathrm{kg} / \mathrm{fed})$, harvest index $(\%)$ and kernels protein yield $(\mathrm{kg} / \mathrm{fed})$ (Tables, 2 and 3). The results in Table, 4 showed that Gemmeiza 9 which fertilized by $112.5 \mathrm{~kg} \mathrm{~N} / \mathrm{fed}$ recorded significantly the highest values of biological yield (6816.66 and $7216.66 \mathrm{~kg} / \mathrm{fed}$ ), grain yield $(2863.33$ and $3013.33 \mathrm{~kg} / \mathrm{fed}$ ) and kernels protein yield ( 335.72 and $353.83 \mathrm{~kg} / \mathrm{fed}$ ) in the first and second seasons, respectively, Whereas, Giza 168 under the same nitrogen fertilizer rate gave the greatest straw yield $(4360.00$ and $4683.33 \mathrm{~kg} / \mathrm{fed}$ ) in the first and second seasons, respectively. Similar results were also reported by Allam 2005; Abd ElMaaboud et al., 2006; Benin et al., 2012; Gomaa et al., 2012; Hafez et al., 2012; Zaki et al., 2012; Ashmawy et al., 2013; Noureldin et al., 2013; Sultana et al., 2013; Khalid et al., 2014; El-Hosary et al., 2015, Jelic et al., 2015 and Mehasen et al., 2015.

\section{The interaction effect between phosphorous fertilizer rates and wheat cultivars:}

The interaction effect between phosphorous fertilizer rates and wheat cultivars were significant on No. of tillers $/ \mathrm{m}^{2}$, No. of spikes $/ \mathrm{m}^{2}$, plant height $(\mathrm{cm})$, biological yield $(\mathrm{kg} / \mathrm{fed})$, grain yield $(\mathrm{kg} / \mathrm{fed})$, straw yield $(\mathrm{kg} / \mathrm{fed})$ and kernels protein yield $(\mathrm{kg} / \mathrm{fed})$ (Tables, 2 and 3 ). The results in Table, 4 showed that Gemmeiza 9 which fertilized by $30 \mathrm{~kg} \quad \mathrm{P}_{2} \mathrm{O}_{5}$ fed recorded significantly the greatest values of biological yield (5760.00 and $6247.50 \mathrm{~kg} / \mathrm{fed}$ ), grain yield (2477.50 and $2672.50 \mathrm{~kg} / \mathrm{fed}$ ) and kernels protein yield (282.44 and 305.27 $\mathrm{kg} / \mathrm{fed}$ ) in the first and second seasons, respectively, Whereas, Giza 168 under the same phosphorous fertilizer rate gave the maximum straw yield (3687.50 and 4112.50 $\mathrm{kg} / \mathrm{fed}$ ) in the first and second seasons, respectively. Similar results were also reported by Abdul Galil et al., 2003; Abd ElMaaboud et al., 2006 and Jelic et al., 2015.

\section{The interaction effect between nitrogen, phosphorous and wheat cultivars:}

Significant effect of the interaction between nitrogen and phosphorous fertilizer rates as well as wheat cultivars obtained for some growth, yield and yield components of wheat namely, No. of tillers $/ \mathrm{m}^{2}$, No. of spikes $/ \mathrm{m}^{2}$, plant height $(\mathrm{cm})$, biological yield $(\mathrm{kg} / \mathrm{fed})$, grain yield $(\mathrm{kg} / \mathrm{fed})$, straw yield $(\mathrm{kg} / \mathrm{fed})$ and kernels protein yield $(\mathrm{kg} / \mathrm{fed})$ in the both seasons (Tables, 2 and 3 ). The results illustrated in Table, 5 indicated that planting Gemmeiza 9 wheat cultivar which fertilized by $112.5 \mathrm{~kg} \mathrm{~N}$ and $30 \mathrm{~kg} \mathrm{P}_{2} \mathrm{O}_{5} / \mathrm{fed}$ recorded the maximum values of biological yield (6970.00 and $7450.00 \mathrm{~kg} / \mathrm{fed}$ ), grain yield (2920.00 and $3150.00 \mathrm{~kg} / \mathrm{fed}$ ) and kernels protein yield (343.70 and 372.57 $\mathrm{kg} / \mathrm{fed}$ ). Whereas, Giza 168 under the same nitrogen and phosphorous fertilizer rates gave the highest straw yield $(4480.00$ and $4850.00 \mathrm{~kg} / \mathrm{fed}$ ) in the first and second seasons, respectively. Such results are in accordance with those obtained by Abd ElMaaboud et al., 2006 and Jelic et al., 2015. 
Table, 4: Effect of the interaction between (nitrogen $X$ phosphorous), (nitrogen $X$ cultivars) and (phosphorous $X$ cultivars) on straw yield $\mathrm{kg} / \mathrm{fed}$, grain yield $\mathrm{kg} / \mathrm{fed}$, biological yield $\mathrm{kg} / \mathrm{fed}$ and kernels protein yield $\mathrm{kg} / \mathrm{fed}$ of wheat during $2011 / 2012$ and $2012 / 2013$ seasons.

\begin{tabular}{|c|c|c|c|c|c|c|c|c|c|}
\hline \multirow{2}{*}{\multicolumn{2}{|c|}{ Treatments }} & \multicolumn{2}{|c|}{ Straw yield kg/fed } & \multicolumn{2}{|c|}{ Grain yield kg/fed } & \multicolumn{2}{|c|}{$\begin{array}{c}\text { Biological yield } \\
\mathrm{kg} / \mathrm{fed}\end{array}$} & \multicolumn{2}{|c|}{ Protein yield kg/fed } \\
\hline & & $2011 / 2012$ & $2012 / 2013$ & $2011 / 2012$ & $2012 / 2013$ & $2011 / 2012$ & $2012 / 2013$ & $2011 / 2012$ & $2012 / 2013$ \\
\hline $\mathrm{N}$ & $\mathrm{P}$ & \multicolumn{8}{|c|}{ The interaction effect between nitrogen and phosphorous fertilization } \\
\hline \multirow{3}{*}{0} & 0 & 2326.67 & 2566.67 & 1153.33 & 1325.00 & 3480.00 & 3891.67 & 121.68 & 141.31 \\
\hline & 15 & 436.67 & 33 & 1446.67 & 1556.67 & 3883.34 & 4300.00 & 155.68 & 41 \\
\hline & 30 & 67 & 33 & 15 & .67 & 4118.34 & 4555.00 & .99 & 40 \\
\hline \multirow{3}{*}{37.5} &  & 070.00 & 67 & 184 & 1993.33 & 4913.33 & 5400.00 & 10 & 60 \\
\hline & 15 & 67 & 33 & 20 & 33 & 5281.67 & 5791.66 & .03 & 90 \\
\hline & 30 & 7 & 00 & 21 & 33 & 5470.00 & 6133.33 & 09 & 52 \\
\hline \multirow{3}{*}{75} &  & 7 & 7 & 7 & 67 & 5743.34 & 6373.34 & 52 & 10 \\
\hline & 15 & 00 & 33 & 23 & 26 & 5988.33 & 6670.00 & 0 & 50 \\
\hline & 30 & 67 & 00 & 24 & 26 & 6170.00 & 6843.33 & 62 & 86 \\
\hline \multirow{3}{*}{112.5} & 0 & 33 & 33 & 246 & 67 & 645 & 6970.00 & 3 & 87 \\
\hline & 15 & 00 & 67 & 257 & 27 & 672 & 7210.00 & 97 & 97 \\
\hline & 30 & 33 & 37 & 262 & 33 & 6873 & 7390.00 & & 12 \\
\hline \multicolumn{2}{|c|}{ L.S.D. at $5 \%$} & 103.52 & 38.92 & 92.50 & 32 & 246.30 & 285.04 & 5 & \\
\hline $\mathrm{N}$ & V & \multicolumn{8}{|c|}{ The interaction effect between nitrogen fertilization and wheat cultivars } \\
\hline \multirow{3}{*}{0} & Gemmeiza 9 & 2240.00 & 2483.33 & 1600.00 & 1745.00 & 3840.00 & 4228.33 & 166.90 & 183.41 \\
\hline & Sakha 93 & 67 & .00 & 00 & 67 & 3906.67 & 4216.67 & .09 & 3.76 \\
\hline & Giza 168 & .33 & 00 & 67 & 67 & 3735.00 & 4301.67 & 70 & .81 \\
\hline \multirow{3}{*}{37.5} & Gemmeiza 9 & .33 & 67 & 00 & 67 & 33 & 5838.34 & 84 & 49 \\
\hline & Sakha 93 & 33 & 67 & 67 & 33 & .00 & 5670.00 & 88 & 65 \\
\hline & & & 67 & 00 & .00 & 511 & 5816.67 & 08 & 47 \\
\hline \multirow{3}{*}{75} & (1) & 00 & 37 & 67 & 33 & 6156.67 & 6670.00 & 65 & .03 \\
\hline & Sakha 93 & 550.00 & 3923.33 & .00 & 2616.67 & 5910.00 & 6540.00 & .02 & .46 \\
\hline & Giza 168 & 853.33 & 4370.00 & 1981.67 & 2306.67 & 5835.00 & 6676.67 & .16 & .87 \\
\hline \multirow{3}{*}{112.5} & Gemmeiza 9 & 3953.33 & 4203.33 & 2863.33 & 3013.33 & 6816.66 & 7216.66 & 5.72 & .83 \\
\hline & Sakha 93 & .33 & 4320.00 & 2526.67 & 2860.00 & 6600.00 & 7180.00 & 297.26 & 337.61 \\
\hline & Giza 168 & 4360.00 & 4683.33 & 2260.00 & 2490.00 & 6620.00 & 7173.33 & 0.52 & .32 \\
\hline \multicolumn{2}{|c|}{ L.S.D. at $5 \%$} & 393.04 & 469.88 & 335.04 & 366.56 & 472.30 & 535.02 & 17.00 & 17.52 \\
\hline $\mathrm{P}$ & V & \multicolumn{8}{|c|}{ The interaction effect between phosphorous fertilization and wheat cultivars } \\
\hline \multirow{3}{*}{0} & Gemmeiza 9 & 3022.50 & .50 & 50 & .25 & 5225.00 & 5698.75 & 31 & .61 \\
\hline & Sakha 93 & 3167.50 & 3462.50 & .50 & .50 & 5130.00 & 5595.00 & 92 & .80 \\
\hline & Giza 168 & 3482.50 & 3812.50 & .50 & .00 & 5080.00 & 5682.50 & 93 & 84 \\
\hline \multirow{3}{*}{15} & Gemmeiza 9 & 3187.50 & 3472.50 & 50 & 6.25 & 5595.00 & 6018.75 & 61 & .66 \\
\hline & Sakha 93 & 3315.00 & 3617.50 & .00 & 00 & 5455.00 & 5927.50 & 3.72 & 264.66 \\
\hline & Giza 168 & 3591.25 & 4007.50 & 1763.75 & 2025.00 & 5355.00 & 6032.50 & 205.09 & 237.20 \\
\hline \multirow{3}{*}{30} & Gemmeiza 9 & 3282.50 & 3575.00 & 2477.50 & 2672.50 & 5760.00 & 6247.50 & 282.44 & 305.27 \\
\hline & Sakha 93 & 3450.00 & 3712.50 & 2222.50 & 00 & 5672.50 & 6182.50 & 254.63 & 284.96 \\
\hline & Giza 168 & 3687.50 & .50 & 3.75 & 2148.75 & 5541.25 & 6261.25 & 217.24 & 253.53 \\
\hline \multicolumn{2}{|c|}{ L.S.D. at $5 \%$} & 340.38 & 406.93 & 290.15 & 317.45 & 409.02 & 463.34 & 14.72 & 15.18 \\
\hline
\end{tabular}


El-Balasy, et al.,

Table, 5: Effect of the interaction between nitrogen, phosphorous and cultivars on straw yield $\mathrm{kg} / \mathrm{fed}$, grain yield $\mathrm{kg} / \mathrm{fed}$, biological yield $\mathrm{kg} / \mathrm{fed}$ and kernels protein yield $\mathrm{kg} / \mathrm{fed}$ of wheat during 2011/2012 and 2012/2013 seasons.

\begin{tabular}{|c|c|c|c|c|c|c|c|c|c|c|}
\hline \multicolumn{3}{|c|}{ Treatments } & \multicolumn{2}{|c|}{ Straw yield kg/fed } & \multicolumn{2}{|c|}{ Grain yield kg/fed } & \multicolumn{2}{|c|}{$\begin{array}{c}\text { Biological yield } \\
\mathrm{kg} / \mathrm{fed}\end{array}$} & \multicolumn{2}{|c|}{$\begin{array}{l}\text { Protein yield } \\
\text { kg/fed }\end{array}$} \\
\hline $\mathrm{N}$ & $P$ & $\mathrm{~V}$ & $2011 / 2012$ & $2012 / 2013$ & $2011 / 2012$ & $2012 / 2013$ & $2011 / 2012$ & $2012 / 2013$ & $2011 / 2012$ & $2012 / 2013$ \\
\hline \multirow{9}{*}{0} & \multirow{3}{*}{0} & eiza 9 & 2090.00 & 2400.00 & 1360.00 & 1525.00 & 3450.00 & 3925.00 & 138.22 & 156.73 \\
\hline & & Sakha 93 & 2280.00 & 2490.00 & 1250.00 & 1300.00 & 3530.00 & 3790.00 & 132.88 & 138.94 \\
\hline & & Giza 168 & 2610.00 & 2810.00 & 850.00 & 1150.00 & 3460.00 & 3960.00 & 92.30 & 126.84 \\
\hline & \multirow{3}{*}{15} & Gemmeiza 9 & 2280.00 & 2490.00 & 1690.00 & 1800.00 & 3970.00 & 4290.00 & 175.61 & 188.07 \\
\hline & & Sakha 93 & 2400.00 & 2710.00 & 1530.00 & 1550.00 & 3930.00 & 4260.00 & 164.39 & 167.42 \\
\hline & & Giza 168 & 2630.00 & 3030.00 & 1120.00 & 1320.00 & 3750.00 & 4350.00 & 124.81 & 147.85 \\
\hline & \multirow{3}{*}{30} & Gemmeiza 9 & 2350.00 & 2560.00 & 1750.00 & 1910.00 & 4100.00 & 4470.00 & 188.03 & 206.31 \\
\hline & & Sakha 93 & 2570.00 & 2780.00 & 1690.00 & 1820.00 & 4260.00 & 4600.00 & 183.51 & 200.74 \\
\hline & & Giza 168 & 2780.00 & 3100.00 & 1215.00 & 1495.00 & 3995.00 & 4595.00 & 136.78 & 169.15 \\
\hline \multirow{9}{*}{37.5} & \multirow{3}{*}{0} & Gemmeiza 9 & 2800.00 & 3160.00 & 2100.00 & 2270.00 & 4900.00 & 5430.00 & 232.82 & 252.96 \\
\hline & & Sakha 93 & 3090.00 & 3330.00 & 1890.00 & 1990.00 & 4980.00 & 5320.00 & 207.38 & 221.76 \\
\hline & & Giza 168 & 3320.00 & 3730.00 & 1540.00 & 1720.00 & 4860.00 & 5450.00 & 174.24 & 195.59 \\
\hline & \multirow{3}{*}{15} & Gemmeiza 9 & 3030.00 & 3340.00 & 2350.00 & 2505.00 & 5380.00 & 5845.00 & 267.23 & 286.28 \\
\hline & & Sakha 93 & 3220.00 & 3510.00 & 2100.00 & 2170.00 & 5320.00 & 5680.00 & 238.80 & 249.24 \\
\hline & & Giza 168 & 3475.00 & 3900.00 & 1670.00 & 1950.00 & 5145.00 & 5850.00 & 192.57 & 228.52 \\
\hline & \multirow{3}{*}{30} & Gemr & 3150.00 & 354 & .00 & 0 & 5600.00 & .00 & 280.00 & 308.88 \\
\hline & & Sakha 93 & 3300.00 & 3620.00 & 2180.00 & 2390.00 & 5480.00 & 6010.00 & 250.38 & 275.87 \\
\hline & & Giza 168 & 3590.00 & 4000.00 & 1740.00 & 2150.00 & 5330.00 & 6150.00 & 203.81 & 253.07 \\
\hline \multirow{9}{*}{75} & \multirow{3}{*}{0} & Gemmeiza 9 & 3350.00 & 37 & 00 & 0 & 0 & 6 & 7 & 310.33 \\
\hline & & Sakha 93 & 3410.00 & 3820.00 & 2280.00 & 2450.00 & 5690.00 & 6270.00 & 262.00 & 283.63 \\
\hline & & Giza 168 & 3790.00 & 4200.00 & 1850.00 & 2210.00 & 5640.00 & 6410.00 & 217.86 & 260.63 \\
\hline & \multirow{3}{*}{15} & Gem & 3480.00 & 3850.00 & .00 & .00 & 6200.00 & 0.00 & 41 & 333.58 \\
\hline & & Sakha 93 & 3540.00 & 3900.00 & 2380.00 & 2640.00 & 5920.00 & 6540.00 & 277.42 & 309.24 \\
\hline & & Giza 168 & 3870.00 & 4410.00 & 1975.00 & 2320.00 & 5845.00 & 6730.00 & 233.59 & 275.72 \\
\hline & \multirow{3}{*}{30} & Gemmeiza 9 & 3580.00 & 3900.00 & 2790.00 & 2930.00 & 6370.00 & 6830.00 & 325.22 & 340.53 \\
\hline & & Sakha 93 & 3700.00 & 4050.00 & 2420.00 & 2760.00 & 6120.00 & 6810.00 & 282.91 & 323.92 \\
\hline & & Giza 168 & 3900.00 & 4500.00 & 2120.00 & 2390.00 & 6020.00 & 6890.00 & 250.98 & 285.40 \\
\hline \multirow{9}{*}{112.5} & \multirow{3}{*}{0} & Gemmeiza 9 & 3850.00 & 4100.00 & 2800.00 & 2900.00 & 6650.00 & 7000.00 & 34 & 337.38 \\
\hline & & Sakha 93 & 3890.00 & 4210.00 & 2430.00 & 2790.00 & 6320.00 & 7000.00 & 284.92 & 328.08 \\
\hline & & Giza 168 & 4210.00 & 4510.00 & 2150.00 & 2400.00 & 6360.00 & 6910.00 & 254.78 & 287.14 \\
\hline & \multirow{3}{*}{15} & Gemmeiza 9 & 3960.00 & 4210.00 & 2870.00 & 2990.00 & 6830.00 & 7200.00 & 336.18 & 351.94 \\
\hline & & Sakha 93 & 4100.00 & 4350.00 & 2550.00 & 2880.00 & 6650.00 & 7230.00 & 300.15 & 340.63 \\
\hline & & Giza 168 & 4390.00 & 4690.00 & 2290.00 & 2510.00 & 6680.00 & 7200.00 & 274.77 & 302.59 \\
\hline & \multirow{3}{*}{30} & Gemmeiza 9 & 4050.00 & 4300.00 & 2920.00 & 3150.00 & 6970.00 & 7450.00 & 343.70 & 372.57 \\
\hline & & Sakha 93 & 4230.00 & 4400.00 & 2600.00 & 2910.00 & 6830.00 & 7310.00 & 306.63 & 344.35 \\
\hline & & Giza 168 & 4480.00 & 4850.00 & 2340.00 & 2560.00 & 6820.00 & 7410.00 & 282.10 & 311.54 \\
\hline \multicolumn{3}{|c|}{ L.S.D. at $5 \%$} & 680.76 & 813.86 & 580.31 & 634.90 & 818.05 & 926.68 & 29.44 & 30.35 \\
\hline
\end{tabular}




\section{CONCLUSION}

From the obtained results of this study it could be concluded that planting wheat Gemmeiza 9 cultivar with fertilizing by $30 \mathrm{~kg}$ $\mathrm{P}_{2} \mathrm{O}_{5}$ with $112.5 \mathrm{~kg} \mathrm{~N} / \mathrm{fed}$ in order to maximizing its productivity.

\section{REFERENCES}

A.O.A.C. (1990). Official methods of analysis association of official analysis chemists, $13^{\text {th }}$ Ed., Washington, D. C., U. S. A.

Abd El-Maaboud, M. Sh., T. E. Khaled and E. Farag (2006). Effect of mineral and biological nitrogen and phosphorous fertilization on some wheat cultivars under, Salinity conditions at Ras Sudr. J. Agric. Sci. Mansoura Univ., 31 (11): 6839-6853.

Abdul Galil, A. A., H. A. Basha, S. A. E. Mowafy and S. M. Mohamed (2003). Effect of phosphorous addition on the response of four wheat cultivars to $\mathrm{N}$ fertilization levels under sandy soil conditions. Minufiya J. Agric. Res., 28 (1): 1-22.

Al-Afandy, K.T., A. A. Abdel-Ati and M. A. Mohamed (2007). Effect of nitrogen, phosphorous and seeding rates on wheat production and weed control in Siwa Oasis. J. Agric. Sci, Mansoura Univ., 32 (8): 6099-6111.

Allam, S. A. (2005). Growth and productivity performance of some wheat cultivars under various nitrogen fertilization levels. J. Agric. Sci, Mansoura Univ., 30 (4): 1871-1880.

Ashmawy, F., M. S. EL-Habal, H. S. Saudy and I. K. Abbas (2013). The relative contribution of yield components to grain yield of some wheat cultivars grown under different nitrogen fertilizer levels. Egypt. J. Agric. Res., 88 (1): 225-239.

Benin, G., E. Bornhofen, E. Beche, E. S. Pagliosa, C. L. Silva and C. Pinnow (2012). Agronomic performance of wheat cultivars in response to nitrogen fertilization levels. Acta Scientiarum. Agronomy, 34(3): 275-283.
Black, C.A. (1965). Methods of Soil Analysis. Amer. Soc. of Agronomy, Inc. Pub. Madison, Wisconsin, USA.

Bulletin of the Agricultural Statistics. Part (1). winter crops, 2014/2015. (2016). Econ. Aff. Sec., Min Agric. \& land Rec., Arab Repu. Egypt.

El-Hosary, A. A., G. Y. M. Hammam, El. M. M. El-Gedwy and M. E. E. Sidi (2015). Response of some wheat cultivars to some organic and mineral nitrogen fertilizer levels. J. Plant Production, Mansoura Univ., 6 (9): 1517-1529.

Gomaa, M. A., N. M. Zaki, F. I. Radwan, M. S. Hassanein, A. M. Gomaa and A. M. Wali (2012). The combined effect of mineral, organic and bio-fertilizers on growth of some wheat cultivars. J. Appl. Sci. Res., 7(11): 1591-1608.

Gomez, K.A. and A.A. Gomez (1984). Statistical procedures for agricultural research. $2^{\text {nd }}$, (ed.). John Wiley and Sons, NY, U.S.A.

Hafez, E. M., H. Aboukhadrah, S. Gh. R. Sorour and A. R. Yousef (2012). Comparison of agronomical and physiological nitrogen use efficiency in three cultivars of wheat as affected by different levels of $n$-sources. Proc. $13^{\text {th }}$ international Conf. Agron., Fac. of Agic., Benha Univ., Egypt, 9-10 September, 130-145.

Jackson, M.L. (1973). Soil chemical analysis. Prentice Hall of India Private limited New Delhi.

Jelic, M., J. Milivojevic, O. Nikolic, V. Djekic and S. Stamenkovic (2015). Effect of long term fertilization and soil amendments on yield, grain quality and nutrition optimization in winter wheat on an acidic Pseudogley. Romanian Agric. Res., 165: 1-174.

Khalid, M. S., M. F. Saleem, S. Ali, M. W. Pervez, M. Rehman, S. Hussain and K. Rehman (2014). Optimization of nitrogen fertilizer level for newly evolved wheat (Triticum aestivum, L.) cultivars. App. Sci. Report. 7 (2): 83-87. 
Khan, P., M. Imtiaz, M. Aslam, S. K. H. Shah, M. Y. Memon and S. Siddiqui (2008). Effect of different nitrogen and phosphorous ration the performance of wheat cultivar (Khirman). Sarhad J. Agric., 24 (2): 233-240.

Mehasen, S. A. S., S. A. Badawy and S. Sh. Abdullah (2015). Influence of bio and mineral nitrogen fertilizers on productivity of some bread wheat varieties. J. Food, Agric. \& Env., 13 (2): 162-167.

Michigan State University (1983). MSTAT-C: Micro- computer Statistical Program, Version 2. Michigan State University, East Lansing.

Noureldin, N. A., H. S. Saudy, F. Ashmawy and H. M. Saed (2013). Grain yield response index of bread wheat cultivars as influenced by nitrogen levels. Annals
Agric. Sci., Ain Shams Univ., Cairo 58 (2): 147-152.

Shafshak, S. E., G. Y. Hammam, S. A. S. Mehasen and S. A. H. Mohamed (2003). Effect of farm yard manure, mineral $\mathrm{N}$ and $\mathrm{P}$ fertilizer on wheat yield. Annals of Agric. Sc., Moshtohor, 41 (4): 1433-1448. Sultana, S. R., A. Ahmad, A. Wajid and J. Akhtar (2013). Estimating growth and yield related traits of wheat genotypes under variable nitrogen application in semi-arid conditions. Pak. J. life Soc. Sci., 11(2): 118-125.

Zaki, N. M., M. A. Gomaa, F. I. Radwan, M. S. Hassanein and A. M. Wali (2012). Effect of mineral, organic and biofertilizers on yield, yield components and chemical composition of some wheat cultivars. J. Appl. Sci. Res., 8(1): 174191. 


\section{تأثير السماد النيتروجيني والفوسفاتي على إنتاجية بعض أصناف القمح}

محمد محمود البلاصي(1) ، علي عبد المقصود الحصري(2)، جابر يحيى همام(2) ، صلاح عباس علام(2)، رأفت بلر أبو عرب(1) ، السعيذ محمد الجدوي(2) ، أحمد علي الحصري(2)

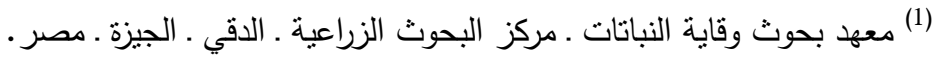
(2) قفم الدحاصيل ـ كلية الزراعة ـ جامعة بنها.

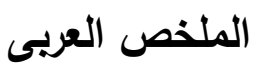

أجريت تجربتان حقليتان في مركز سيدي سالم . محافظة كفر الثيخ · جمهورية مصر العربية، خلال الموسمين الثتوين

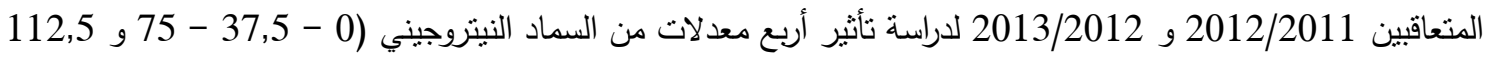

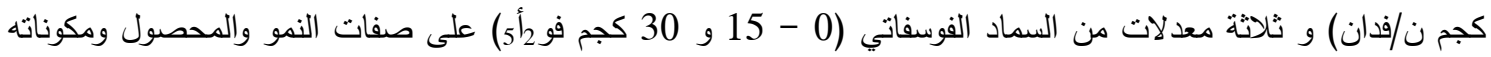

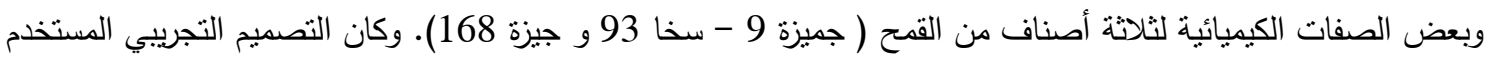
هو قطع منشقة مرتان في أربع مكررات.

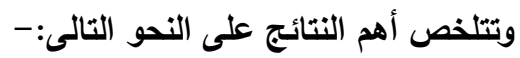

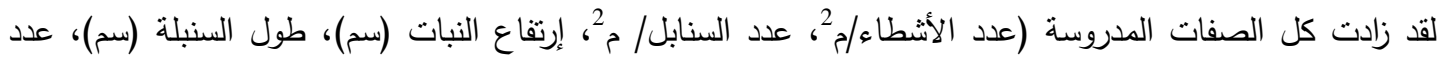
حبوب السنبلة، وزن 1000 حبة (جم) وزن حبوب السنبلة (جم)، الدحصول البيولوجي كجم/فدان، محصول الحبوب

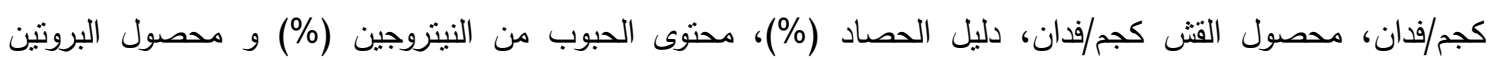
كجم/فدان في كلا الموسمين) معنويا بزيادة معدلات السماد النيتروجيني ما عدا محتوى الحبوب من الفوسفور لم لمتأثرئ معنويا. إضافة السماد النيتروجيني بمعدل 112,5 كجم نبتروجين /فدان أعطى أفضل قيم في الصفات الصنات السابقة.


النبات، طول السنبلة، المحصول البيولوجي/فدان، محصول الحبوب/فدان، محصول القش/فدان و محتوى الحبوب من الفوسفور في كلا الموسمين، بينما عدد حبوب السنبلة، وزن 1000 حبة، وزن حبوب السنبلة، دليل الحصاد، محتوى الحبوب من النيتروجين و محصول البروتين/فدان لم تتأثز معنويا زيادة السماد الفوسفاتي.

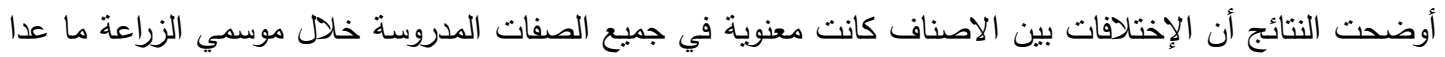

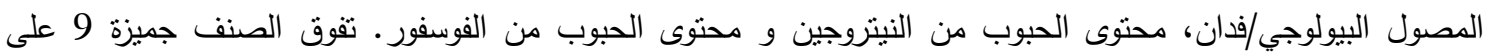



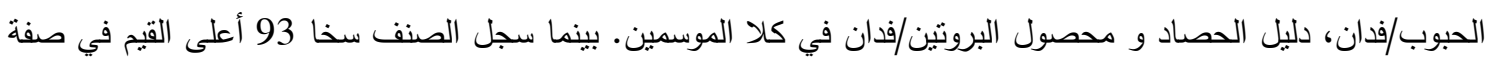

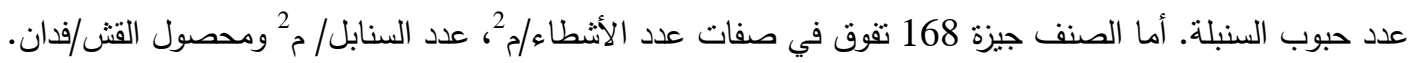

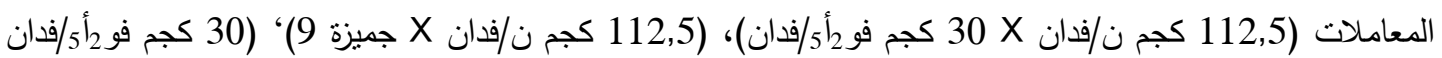

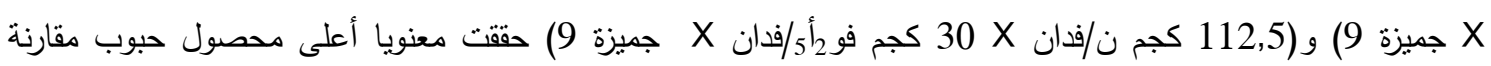
بالمعاملات الأخري في كلا الموسمين. توصي النتائج بزراعة الصنف جميزة 9 والتسميد بالسماد الفوسفاتي بمعدل 30 كجم فو وأك/فدان و 112,5 كجم ن/فدان 